\title{
Effectiveness of computer-aided diagnosis of colorectal lesions using novel software for magnifying narrow-band imaging: a pilot study
}

\section{(ㄷ)(i) $\odot$}

\author{
Authors

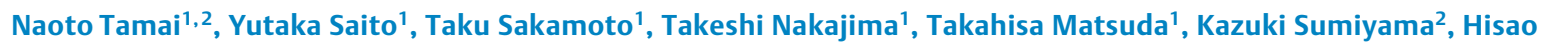 \\ Tajiri $^{3}$, Ryosuke Koyama ${ }^{4}$, Shoji Kido ${ }^{4}$
}

Institutions

1 Endoscopy Division, National Cancer Center Hospital, Tokyo, Japan

2 Department of Endoscopy, The Jikei University School of Medicine, Tokyo, Japan

3 Department of Innovative Interventional Endoscopy Research, The Jikei University School of Medicine, Tokyo, Japan

4 Applied Medical Engineering Science, Graduate School of Medicine, Yamaguchi University, Yamaguchi, Japan

submitted 12.10.2016

accepted after revision $\quad 6.2 .2017$

\author{
Bibliography \\ DOI https://doi.org/10.1055/s-0043-105490 | \\ Endoscopy International Open 2017; 05: E690-E694 \\ (c) Georg Thieme Verlag KG Stuttgart · New York \\ ISSN 2364-3722
}

Corresponding author

Naoto Tamai, MD, PhD, Department of Endoscopy, The Jikei University School of Medicine, 3-25-8 Nishi Shinbashi, Minato-ku, Tokyo 105-8461, Japan

Phone: +81-3-3433-1111 (Ext. 3181)

Fax: +81-3-3459-4524

tamai-naoto@jikei.ac.jp

\section{ABSTRACT}

Background and study aims Magnifying narrow-band imaging (M-NBI) enables detailed observation of microvascular architecture and can be used in endoscopic diagnosis of colorectal lesion. However, in clinical practice, differential diagnosis and estimation of invasion depth of colorectal lesions based on $\mathrm{M}-\mathrm{NBI}$ findings require experience. Therefore, developing computer-aided diagnosis (CAD) for M-NBI would be beneficial for clinical practice. The aim of this study was to evaluate the effectiveness of software for CAD of colorectal lesions.

Materials and methods In collaboration with Yamaguchi University, we developed novel software that enables CAD of colorectal lesions using M-NBI images. This software for CAD further specifically divides original Sano's colorectal $\mathrm{M}-\mathrm{NBI}$ classification into 3 groups (group A, capillary pattern [CP] type I; group B, CP type II + CP type IIIA; group C, CP type IIIB), which describe hyperplastic polyps (HPs), adenoma/adenocarcinoma (intramucosal [IM] to submucosal [SM]-superficial) lesions, and SM-deep lesions, respectively. We retrospectively reviewed 121 lesions evaluated using M-NBI.

Results The 121 reviewed lesions included $21 \mathrm{HP}, 80$ adenoma/adenocarcinoma (IM to SM-superficial), and $20 \mathrm{SM}$ deep lesions. The concordance rate between the CAD and the diagnosis of the experienced endoscopists was $90.9 \%$. The sensitivity, specificity, positive and negative predictive values, and accuracy of the CAD for neoplastic lesions were $83.9 \%, 82.6 \%, 53.1 \%, 95.6 \%$, and $82.8 \%$, respectively. The values for SM-deep lesions were $83.9 \%, 82.6 \%$, 53.1\%, $95.6 \%$, and $82.8 \%$, respectively.

Conclusion Relatively high diagnostic values were obtained using CAD. This software for CAD could possibly lead to a wider use of $\mathrm{M}-\mathrm{NBI}$ in the endoscopic diagnosis of colorectal lesions.

\section{Introduction}

Early detection and removal of colorectal neoplasia are essential in reducing mortality rates for patients with colorectal cancer $[1,2]$. Although several modalities are available for colorectal cancer screening [3-6], colonoscopy is considered the most effective because it allows direct visualization and on- site treatment of encountered lesions. Accurately determining the histological features of colorectal polyps using endoscopy could prevent unnecessary endoscopic treatments thus allowing the proposal of adequate surveillance recommendations [7]. Magnifying narrow-band imaging (NBI) enables detailed observation of the microvascular architecture of lesions and can be used for endoscopic differential diagnosis and estima- 


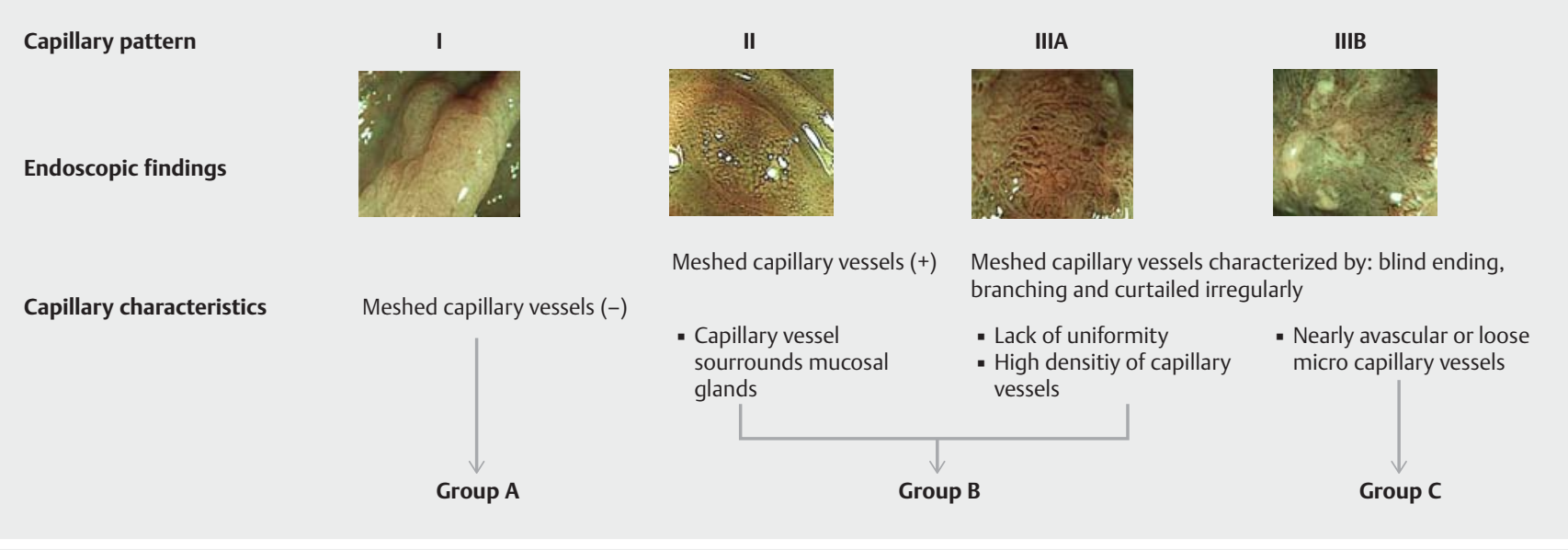

- Fig. 1 Sano's classification (modified for this study). In this study, Sano's classification was further divided broadly into three groups: group A, capillary pattern (CP) type I; group B, CP type II + CP type IIIA; group C, CP type IIIB.

tion of invasion depth of colorectal lesions [8,9]. We have developed software for computer-aided diagnosis (CAD) of colorectal lesions into nonneoplastic lesions (hyperplastic polyps [HPs]), adenoma/intramucosal (IM) cancers/submucosal (SM)superficial cancers (invasion depth, $<1000 \mu \mathrm{m}$ ), or SM-deep cancers (invasion depth, $\geq 1000 \mu \mathrm{m}$ ). The aim of this study was to retrospectively evaluate the effectiveness of CAD of colorectal lesions using our novel software and still images obtained from M-NBI. The aim of this study is to evaluate the effectiveness of CAD of colorectal lesions using novel software.

\section{Materials and methods}

\section{Software for CAD}

Using class-appropriate images obtained with front-view M-NBI, we determined the region of interest (ROI), converted the images into grayscale images, visualized the rough vessels through binarization using the moving average method, deleted all colors except the color of the vessel, and removed isolated points and holes. Subsequently, we used characteristics, such as the average width, length, total length, and concentration of the visualized vessel, and the fractal dimension to extract significant quantitative characteristics. Then, we extracted the average length, total length, and concentration of the visualized vessel as the effective quantitative characteristics for classifying colorectal lesions. In corroboration with Yamaguchi University, we developed software for CAD of lesions using the 3 quantitative characteristics. This software for CAD further broadly divides Sano's classification, which is the original colorectal NBI classification [10-12], into 3 groups (group $A$, capillary pattern [CP] type I; group $B, C P$ type II $+C P$ type IIIA; group C, CP type IIIB; $>$ Fig. 1), which describe hyperplastic lesions, adenoma/adenocarcinoma (IM-SM superficial) lesions, and SM-deep lesions, respectively.
The first step in using this software for CAD is to attach the $\mathrm{M}-\mathrm{NBI}$ image to the central window. Subsequently, the ROI to be evaluated is determined using a cursor. Then, by clicking "Calculate" and "Classify" on the software for CADinterface, the M-NBI image can be automatically classified into 1 of 3 groups ( $\triangleright$ Fig. 2 ).

\section{Histological assessment}

All specimens were fixed in $10 \%$ buffered formalin and cut into 2-mm slices. The specimens were then examined microscopically for depth of invasion and classified according to histological type. Histological diagnoses were based on the Japanese classification system of colon and rectal cancers and on the Vienna classification system.

\section{Statistical analysis}

Statistical analysis was performed using SPSS for Windows (SPSS, release 6.0, 1993; SPSS Inc., Chicago, IL, USA). To determine differences in diagnostic accuracies between the CAD and diagnosis of experienced endoscopists for the differentiation and estimation of invasion depth of colorectal neoplasia, the Fisher exact and $X^{2}$ tests were used. A $P$ value $<0.05$ was considered statistically significant.

\section{Method}

We retrospectively reviewed medical records of 103 consecutive patients with 121 lesions that were evaluated using magnifying colonoscopy (CF-H260AZI, CF-FH260AZI; Olympus Medical Systems) and M-NBI before endoscopic or surgical treatment of colorectal neoplasia at the Jikei University Hospital between June 2008 and March 2010. An experienced endoscopist who was blinded to this study selected the most representative $\mathrm{M}-\mathrm{NBI}$ image of each colorectal lesion with the highest magnification and identified the ROI. Then, CAD, using our novel software, was performed on the ROI. Endoscopic diagnosis was also conducted using the selected M-NBI image, with the diagnosis made based on the agreement between 2 experienced endoscopists who were not involved in selection of M-NBI images 


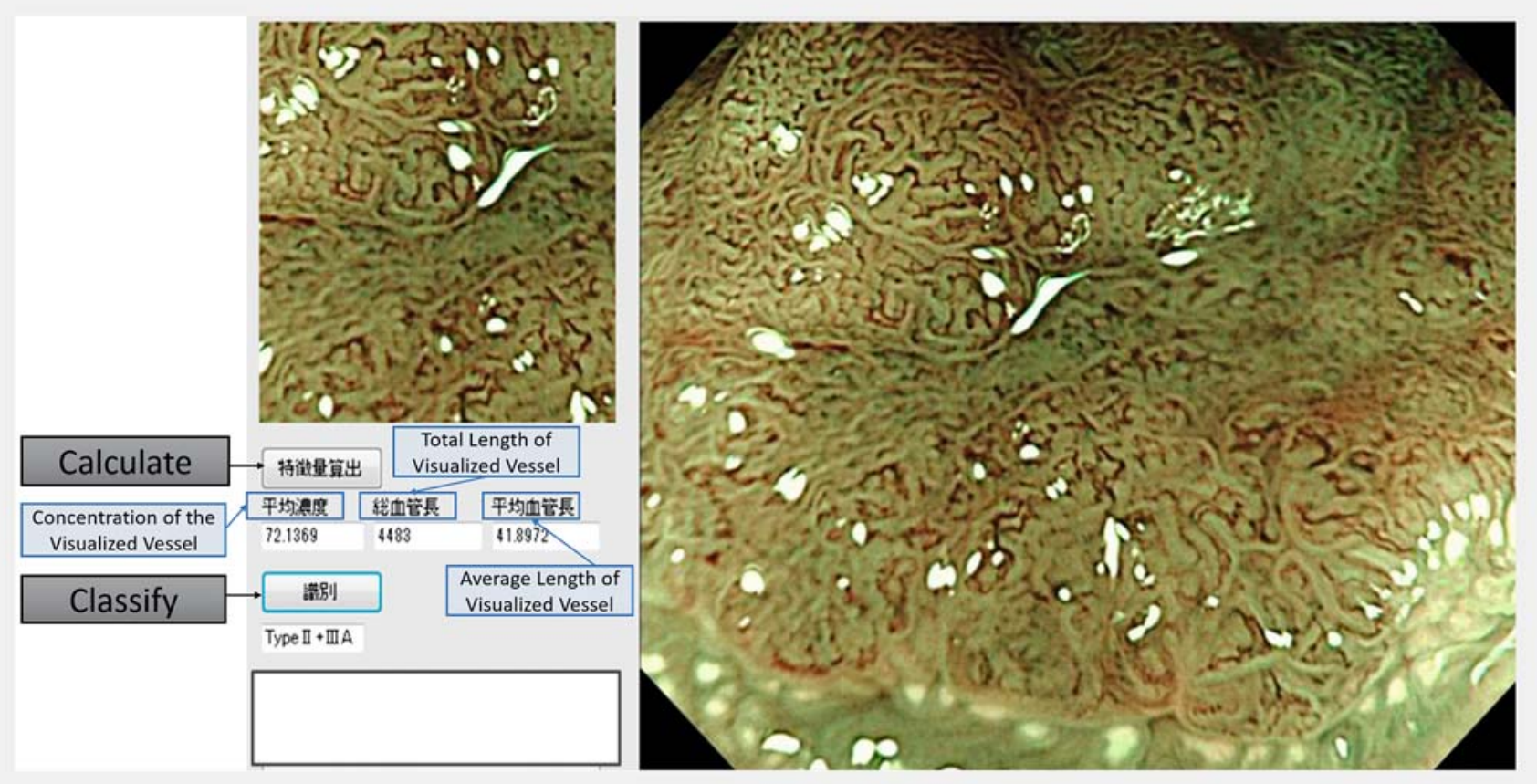

Fig. 2 The user interface of software for computer-aided diagnosis (CAD). The first step when using this software for CAD is to choose the region of interest using the cursor that appears on the attached narrow-band image (right-side window). Then, by clicking "Calculate" and "Classify," the image is automatically classified into 1 of the 3 groups.

and identification of the ROI, and blinded to other endoscopic images of the lesions and the pathological information. All images were classified into the 3 groups described previously (groups A, B, or C). We then compared the results of the CAD using novel software and the diagnoses by the experienced endoscopists to evaluate the effectiveness of the CADusing novel software in the differential diagnosis and estimation of invasion depth of colorectal lesions.

\section{Results}

Based on the histological assessment, the 121 reviewed lesions included $21 \mathrm{HPs}, 80$ adenoma/adenocarcinoma (IM to SM-superficial) lesions, and 20 SM-deep lesions. Among these lesions, 26 lesions were classified into group A, 79 were lesions were classified into group $B$, and 16 lesions were classified into group $C$ by CAD. By contrast, 21 lesions were classified into group A, 82 lesions were classified into group B, and 18 lesions were classified into group $C$ by the experienced endoscopists. The concordance rate between the CAD using our novel software and the diagnoses by the experienced endoscopists was 90.9\% ( $\triangleright$ Table 1 ).

The sensitivity, specificity, positive and negative predictive values (PPV and NPV, respectively), and accuracy of the novel software for CAD in diagnosing neoplastic lesions were $95.0 \%$, $100 \%, 100 \%, 80.8 \%$, and $95.9 \%$, respectively ( Table 2 ). For SM-deep lesions, the rates were 55\%, 95.0\%, 68.8\%, 91.4\%, and $88.4 \%$, respectively ( $\triangleright$ Table 3 ).
- Table 1 Concordance rates between computer-aided diagnosis using software and endoscopists' diagnosis.

\begin{tabular}{|l|c|c|c|}
\hline & Group A (DE) & Group B (DE) & Group C (DE) \\
\hline Group A (DS) & 21 & 5 & 0 \\
\hline Group B (DS) & 0 & 75 & 4 \\
\hline Group C (DS) & 0 & 2 & 14 \\
\hline $\begin{array}{l}\text { DE, diagnosis by the endoscopists; DS, diagnosis using the software for } \\
\text { computer-aided diagnosis. } \\
\text { The concordance rate between computer-aided diagnosis using software } \\
\text { and experienced endoscopists' diagnoses was } 90.9 \%(110 / 121) .\end{array}$ \\
\hline
\end{tabular}

- Table 2 Diagnostic value of the computer-aided diagnosis using software for neoplastic lesions.

\begin{tabular}{|l|c|c|}
\hline & $\begin{array}{l}\text { HP (nonneo- } \\
\text { plastic lesion) }\end{array}$ & $\begin{array}{c}\text { Adenoma/adenocarcinoma } \\
\text { (IM, SM-superficial, and SM- } \\
\text { deep) (neoplastic lesion) }\end{array}$ \\
\hline Group A (DS) & 21 & 5 \\
\hline Group B + C (DS) & 0 & 95 \\
\hline $\begin{array}{l}\text { HP, hyperplastic polyp; IM, intramucosal; SM, submucosal; DS, diagnosis } \\
\text { using the software for computer-aided diagnosis. } \\
\text { The diagnostic accuracy rate of the computer-aided diagnosis using soft- } \\
\text { ware was 95.9\% (116/121) for neoplastic lesions. }\end{array}$
\end{tabular}


- Table 3 Diagnostic value of the computer-aided diagnosis using software for SM-deep lesions.

\begin{tabular}{|l|l|l|}
\hline SM-deep lesions & Non-SM-deep lesions \\
\hline Group A + B (DS) & 9 & 96 \\
\hline Group C (DS) & 11 & 5 \\
\hline $\begin{array}{l}\text { SM, submucosal; DS, diagnosis using the software for computer-aided diag- } \\
\text { nosis. } \\
\text { The diagnostic accuracy rate of the computer-aided diagnosis using soft- } \\
\text { ware was } 88.4 \%(107 / 121) \text { for SM-deep lesions. }\end{array}$ \\
\hline
\end{tabular}

- Table4 Diagnostic value of the endoscopists for neoplastic lesions.

\begin{tabular}{l|l|l|}
$\begin{array}{l}\text { HP (nonneo- } \\
\text { plastic lesion) }\end{array}$ & $\begin{array}{l}\text { Adenoma/adenocarcinoma } \\
\text { (IM to SM-superficial)/SM- } \\
\text { deep }\end{array}$ \\
\hline Group A (DE) & 21 & 0 \\
\hline Group B + C (DE) & 0 & 100 \\
\hline $\begin{array}{l}\text { HP, hyperplastic polyp; IM, intramucosal; SM, submucosal; DE, diagnosis by } \\
\text { the endoscopists. } \\
\text { The diagnostic accuracy rate of the experienced endoscopists was } 100 \% \\
\text { (121/121) for neoplastic lesions. }\end{array}$
\end{tabular}

Table 5 Diagnostic value of the endoscopists for SM-deep lesions.

\begin{tabular}{|l|c|c|}
\hline & SM-deep lesions & Non-SM-deep lesions \\
\hline Group A+B (DE) & 6 & 97 \\
\hline Group C (DE) & 14 & 4 \\
\hline
\end{tabular}

SM, submucosal; DE, diagnosis by the endoscopists.

The diagnostic accuracy rate of the experienced endoscopists was $91.7 \%$

$(111 / 121)$ for SM-deep lesions.

In the diagnoses by experienced endoscopists, all HPs were classified as type $\mathrm{A}$ and all neoplastic lesions were classified as type B or C ( $\triangleright$ Table 4). The sensitivity, specificity, PPV, NPV, and accuracy of their diagnoses of SM-deep lesions were $70.0 \%, 96.1 \%, 77.8 \%, 94.2 \%$, and $92.6 \%$, respectively ( $>$ Table 5).

No significant difference in the accuracies of differential diagnosis of colorectal lesions was observed between the CAD and the endoscopists' diagnosis $(P>0.05)$. Moreover, no significant difference in the diagnostic accuracy of invasion depth was found between the CAD and the endoscopists' diagnosis $(P>0.05)$.

\section{Discussion}

Accurately differentiating between neoplastic and nonneoplastic lesions and estimating the invasion depth of colorectal neoplasia are essential in determining appropriate endoscopic treatment. Currently, magnifying chromoendoscopy with dye (indigo carmine/crystal violet), which has high diagnostic accuracy in differentiating colorectal lesions and in estimating the

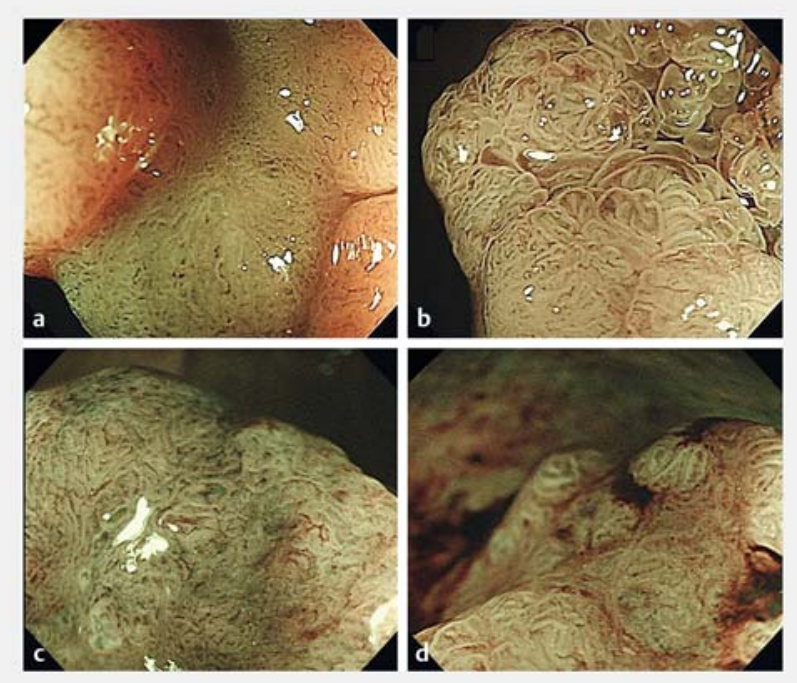

- Fig. 3 Lesions diagnosed differently between the endoscopists and the software for computer-aided diagnosis. a Lesion diagnosed as group $B$ by the endoscopists, and diagnosed as group $C$ by the software for computer-aided diagnosis Macroscopic Type: Is, Pathological diagnosis: Intramucosal adenocarcinoma in tubular adenoma. $\mathbf{b}$ Lesion diagnosed as group B by the endoscopists, and diagnosed as group $C$ by the software for computer-aided diagnosis Macroscopic Type: Is + Ila, Depth of invasion: Intramucosal adenocarcinoma in tubulovillous adenoma. $\mathbf{c}$ Lesion diagnosed as group $C$ by the endoscopists, and diagnosed as group B by the software for computer-aided diagnosis Macroscopic Type: Ip + Ilc, Pathological diagnosis: Intramucosal adenocarcinoma with adenoma component. Depth of invasion: Submucosal Deep (3600 um). d Lesion diagnosed as group $C$ by the endoscopists, and diagnosed as group $B$ by the software for computer-aided diagnosis Macroscopic Type: Is, Depth of invasion: Intramucosal adenocarcinoma in tubular adenoma.

invasion depth of colorectal lesions, should be considered as the standard criterion [13-16]. However, chromoendoscopic observation requires much time, compared not only with white light observation but also with NBI observation [17]. M-NBI enables detailed observation of microvascular architecture and can be used for endoscopic differential diagnosis and estimation of invasion depth of colorectal lesions. However, magnifying observation of colorectal lesions has not been standardized in many Western countries. In addition, use of M-NBI in clinical practice requires knowledge and experience; thus, endoscopic differential diagnosis and estimation of invasion depth of colorectal lesions based on the findings of $\mathrm{M}-\mathrm{NBI}$ has not become widespread in Western countries [18]. Therefore, a simple and automated interpretation using an objective standard of $\mathrm{M}-\mathrm{NBI}$ findings would be beneficial for clinical practice.

The results of this study show that relatively high identification rates were obtained with CAD of M-NBI images for differential diagnosis and estimation of invasion depth of colorectal lesions, and that the diagnostic values were not significantly different from those of experienced endoscopists, although several lesions were diagnosed differently by the endoscopists and CAD using software ( $\triangleright$ Fig. 3 ). In addition, this software for 
CAD can be further improved by strengthening the reliability of each quantitative characteristic and seeking new ones.

This study has several limitations. The number of images used in this study was limited, sample size was not calculated, and the images were obtained from a single center. In addition, determination of ROI was conducted by a single experienced endoscopist, thus precluding determination of interobserver agreement. The 2 lesions diagnosed as Group C by the CAD using software but diagnosed as Group B are presented ( $\triangleright$ Fig. $\mathbf{3} \mathbf{a}, \nabla$ Fig. $\mathbf{3}$ b). One of them had a villous component that often presents blood pooling ( $\boldsymbol{F}$ Fig. $\mathbf{3} \mathbf{b}$ ). Considering this result and the software that uses visualized vessel findings for the diagnosis of the lesions, novel software for CAD might have limitations for diagnosis of a villous lesion.

\section{Conclusion}

With further improvement of the software used in the current study, CAD as aided by this novel software could possibly lead to a wider use of $\mathrm{M}-\mathrm{NBI}$ in the endoscopic diagnosis of colorectal lesions.

\section{Acknowledgment}

This study was supported by Health and Labor Sciences Research Grant (Third Term Comprehensive Control Research for Cancer) from the Ministry of Health, Labour and Welfare of Japan

Competing interests

None

\section{References}

[1] Zauber AG, Winawer SJ, O'Brien M] et al. Colonoscopic polypectomy and long-term prevention of colorectal-cancer deaths. N Engl J Med 2012; 366: 687-696

[2] Shaukat A, Mongin S], Geisser MS et al. Long-term mortality after screening for colorectal cancer. N Engl J Med 2013; 369: 1106-1114

[3] Utano K, Nagata K, Honda T etal. Diagnostic performance and patient acceptance of reduced-laxative CT colonography for the detection of polypoid and non-polypoid neoplasms: a multicenter prospective trial. Radiology 2017; 282: 399-407

[4] Spada C, Pasha SF, Gross SA et al. Accuracy of first- and second-generation colon capsules in endoscopic detection of colorectal polyps: a systematic review and meta-analysis. Clin Gastroenterol Hepatol 2016; DOI: 10.1016/j.cgh.2016.04.038

[5] Saito Y, Saito S, Oka S et al. Evaluation of the clinical efficacy of colon capsule endoscopy in the detection of lesions of the colon: prospective, multicenter, open study. Gastrointest Endosc 2015; 82: 861 869

[6] Kakugawa Y, Saito Y, Matsuda T et al. Colorectal laterally spreading tumors by computed tomographic colonography. Int J Mol Sci 2013; 14: $23629-23638$

[7] Repici A, Hassan C, Radaelli F et al. Accuracy of narrow-band imaging in predicting colonoscopy surveillance intervals and histology of distal diminutive polyps: results from a multicenter, prospective trial. Gastrointest Endosc 2013; 78: 106 - 114

[8] Sano Y, Ikematsu H, Fu KI et al. Meshed capillary vessels by use of narrow-band imaging for differential diagnosis of small colorectal polyps. Gastrointest Endosc 2009; 69: 278-283

[9] Ikematsu H, Matsuda T, Emura F et al. Efficacy of capillary pattern type IIIA/IIIB by magnifying narrow band imaging for estimating depth of invasion of early colorectal neoplasms. BMC Gastroenterol 2010; 10: 33-230X-10-33

[10] Horimatsu T, Sano Y, Kaneko K et al. Relationship between MVD and meshed-capillaries using magnifying NBI colonoscopy in colorectal precursor lesions. Hepatogastroenterology 2009; 56: 372-377

[11] Katagiri A, Fu KI, Sano Y et al. Narrow band imaging with magnifying colonoscopy as diagnostic tool for predicting histology of early colorectal neoplasia. Aliment Pharmacol Ther 2008; 27: 1269-1274

[12] Machida H, Sano Y, Hamamoto Y et al. Narrow-band imaging in the diagnosis of colorectal mucosal lesions: a pilot study. Endoscopy 2004; 36: $1094-1098$

[13] Fu KI, Sano Y, Kato S et al. Chromoendoscopy using indigo carmine dye spraying with magnifying observation is the most reliable method for differential diagnosis between non-neoplastic and neoplastic colorectal lesions: a prospective study. Endoscopy 2004; 36: $1089-$ 1093

[14] Matsuda T, Fujii T, Saito Y et al. Efficacy of the invasive/non-invasive pattern by magnifying chromoendoscopy to estimate the depth of invasion of early colorectal neoplasms. Am J Gastroenterol 2008; 103 : $2700-2706$

[15] Sakamoto T, Saito Y, Nakajima T et al. Comparison of magnifying chromoendoscopy and narrow-band imaging in estimation of early colorectal cancer invasion depth: a pilot study. Dig Endosc 2011; 23: $118-123$

[16] Wanders LK, East JE, Uitentuis SE et al. Diagnostic performance of narrowed spectrum endoscopy, autofluorescence imaging, and confocal laser endomicroscopy for optical diagnosis of colonic polyps: a meta-analysis. Lancet Oncol 2013; 14: 1337-1347

[17] Sakamoto T, Matsuda T, Aoki T et al. Time saving with narrow-band imaging for distinguishing between neoplastic and non-neoplastic small colorectal lesions. J Gastroenterol Hepatol 2012; 27: 351-355

[18] Kiesslich R, Jung M, DiSario JA et al. Perspectives of chromo and magnifying endoscopy: how, how much, when, and whom should we stain? J Clin Gastroenterol 2004; 38: 7-13 